\title{
An Implicit Iteration Method for Variational Inequalities over the Set of Common Fixed Points for a Finite Family of Nonexpansive Mappings in Hilbert Spaces
}

\author{
Nguyen Buong ${ }^{1}$ and Nguyen Thi Quynh Anh ${ }^{2}$ \\ ${ }^{1}$ Vietnamese Academy of Science and Technology, Institute of Information Technology, 18, \\ Hoang Quoc Viet, Cau Giay, Ha Noi 122100, Vietnam \\ ${ }^{2}$ Department of Information Technology, Thai Nguyen National University, \\ Thainguye 842803, Vietnam
}

Correspondence should be addressed to Nguyen Buong, nbuong@ioit.ac.vn

Received 17 December 2010; Accepted 7 March 2011

Academic Editor: Jong Kim

Copyright (C) 2011 N. Buong and N. T. Quynh Anh. This is an open access article distributed under the Creative Commons Attribution License, which permits unrestricted use, distribution, and reproduction in any medium, provided the original work is properly cited.

We introduce a new implicit iteration method for finding a solution for a variational inequality involving Lipschitz continuous and strongly monotone mapping over the set of common fixed points for a finite family of nonexpansive mappings on Hilbert spaces.

\section{Introduction}

Let $C$ be a nonempty closed and convex subset of a real Hilbert space $H$ with inner product $\langle\cdot, \cdot\rangle$ and norm $\|\cdot\|$, and let $F: H \rightarrow H$ be a nonlinear mapping. The variational inequality problem is formulated as finding a point $p^{*} \in C$ such that

$$
\left\langle F\left(p^{*}\right), p-p^{*}\right\rangle \geq 0, \quad \forall p \in C
$$

Variational inequalities were initially studied by Kinderlehrer and Stampacchia in [1] and ever since have been widely investigated, since they cover as diverse disciplines as partial differential equations, optimal control, optimization, mathematical programming, mechanics, and finance (see [1-3]). 
It is well known that if $F$ is an $L$-Lipschitz continuous and $\eta$-strongly monotone, that is, $F$ satisfies the following conditions:

$$
\begin{gathered}
\|F(x)-F(y)\| \leq L\|x-y\|, \\
\langle F(x)-F(y), x-y\rangle \geq \eta\|x-y\|^{2},
\end{gathered}
$$

where $L$ and $\eta$ are fixed positive numbers, then (1.1) has a unique solution. It is also known that (1.1) is equivalent to the fixed-point equation

$$
p=P_{C}(p-\mu F(p))
$$

where $P_{C}$ denotes the metric projection from $x \in H$ onto $C$ and $\mu$ is an arbitrarily fixed positive constant.

Let $\left\{T_{i}\right\}_{i=1}^{N}$ be a finite family of nonexpansive self-mappings of $C$. For finding an element $p \in \cap_{i=1}^{N} \operatorname{Fix}\left(T_{i}\right), \mathrm{Xu}$ and Ori introduced in [4] the following implicit iteration process. For $x_{0} \in C$ and $\left\{\beta_{k}\right\}_{k=1}^{\infty} \subset(0,1)$, the sequence $\left\{x_{k}\right\}$ is generated as follows:

$$
\begin{aligned}
x_{1} & =\beta_{1} x_{0}+\left(1-\beta_{1}\right) T_{1} x_{1}, \\
x_{2} & =\beta_{2} x_{1}+\left(1-\beta_{2}\right) T_{2} x_{2}, \\
& \vdots \\
x_{N} & =\beta_{N} x_{N-1}+\left(1-\beta_{N}\right) T_{N} x_{N}, \\
x_{N+1} & =\beta_{N+1} x_{N}+\left(1-\beta_{N+1}\right) T_{1} x_{N+1},
\end{aligned}
$$

The compact expression of the method is the form

$$
x_{k}=\beta_{k} x_{k-1}+\left(1-\beta_{k}\right) T_{[k]} x_{k}, \quad k \geq 1
$$

where $T_{[n]}=T_{n} \bmod N$, for integer $n \geq 1$, with the mod function taking values in the set $\{1,2, \ldots, N\}$. They proved the following result.

Theorem 1.1. Let $H$ be a real Hilbert space and $C$ a nonempty closed convex subset of $H$. Let $\left\{T_{i}\right\}_{i=1}^{N}$ be $N$ nonexpansive self-maps of $C$ such that $\cap_{i=1}^{N} \operatorname{Fix}\left(T_{i}\right) \neq \emptyset$, where $\operatorname{Fix}\left(T_{i}\right)=\left\{x \in C: T_{i} x=x\right\}$. Let $x_{0} \in C$ and $\left\{\beta_{k}\right\}_{k=1}^{\infty}$ be a sequence in $(0,1)$ such that $\lim _{k \rightarrow \infty} \beta_{k}=0$. Then, the sequence $\left\{x_{k}\right\}$ defined implicitly by (1.5) converges weakly to a common fixed point of the mappings $\left\{T_{i}\right\}_{i=1}^{N}$. 
Further, Zeng and Yao introduced in [5] the following implicit method. For an arbitrary initial point $x_{0} \in H$, the sequence $\left\{x_{k}\right\}_{k=1}^{\infty}$ is generated as follows:

$$
\begin{aligned}
x_{1} & =\beta_{1} x_{0}+\left(1-\beta_{1}\right)\left[T_{1} x_{1}-\lambda_{1} \mu F\left(T_{1} x_{1}\right)\right], \\
x_{2} & =\beta_{2} x_{1}+\left(1-\beta_{2}\right)\left[T_{2} x_{2}-\lambda_{2} \mu F\left(T_{2} x_{2}\right)\right], \\
& \vdots \\
x_{N} & =\beta_{N} x_{N-1}+\left(1-\beta_{N}\right)\left[T_{N} x_{N}-\lambda_{N} \mu F\left(T_{N} x_{N}\right)\right], \\
x_{N+1} & =\beta_{N+1} x_{N}+\left(1-\beta_{N+1}\right)\left[T_{1} x_{N+1}-\lambda_{N+1} \mu F\left(T_{1} x_{N+1}\right)\right],
\end{aligned}
$$

The scheme is written in a compact form as

$$
x_{k}=\beta_{k} x_{k-1}+\left(1-\beta_{k}\right)\left[T_{[k]} x_{k}-\lambda_{k} \mu F\left(T_{[k]} x_{k}\right)\right], \quad k \geq 1 .
$$

They proved the following result.

Theorem 1.2. Let $H$ be a real Hilbert space and $F: H \rightarrow H$ a mapping such that for some constants $L, \eta>0, F$ is L-Lipschitz continuous and $\eta$-strongly monotone. Let $\left\{T_{i}\right\}_{i=1}^{N}$ be $N$ nonexpansive selfmaps of $H$ such that $C=\cap_{i=1}^{N} \operatorname{Fix}\left(T_{i}\right) \neq \emptyset$. Let $\mu \in\left(0,2 \eta / L^{2}\right)$, and let $x_{0} \in H$, with $\left\{\lambda_{k}\right\}_{k=1}^{\infty} \subset[0,1)$ and $\left\{\beta_{k}\right\}_{k=1}^{\infty} \subset(0,1)$ satisfying the conditions: $\sum_{k=1}^{\infty} \lambda_{k}<\infty$ and $\alpha \leq \beta_{k} \leq \beta, k \geq 1$, for some $\alpha, \beta \in(0,1)$. Then, the sequence $\left\{x_{k}\right\}$ defined by (1.7) converges weakly to a common fixed point of the mappings $\left\{T_{i}\right\}_{i=1}^{N}$. The convergence is strong if and only if $\liminf _{k \rightarrow \infty} d\left(x_{k}, C\right)=0$. self-maps.

Recently, Ceng et al. [6] extended the above result to a finite family of asymptotically

Clearly, from $\sum_{k=1}^{\infty} \lambda_{k}<\infty$ we have that $\lambda_{k} \rightarrow 0$ as $k \rightarrow \infty$. To obtain strong convergence without the condition $\sum_{k=1}^{\infty} \lambda_{k}<\infty$, in this paper we propose the following implicit algorithm:

$$
x_{t}=T^{t} x_{t}, \quad T^{t}:=T_{0}^{t} T_{N}^{t} \cdots T_{1}^{t}, \quad t \in(0,1),
$$

where $T_{i}^{t}$ are defined by

$$
T_{i}^{t} x=\left(1-\beta_{t}^{i}\right) x+\beta_{t}^{i} T_{i} x, \quad i=1, \ldots, N, \quad T_{0}^{t} y=\left(I-\lambda_{t} \mu F\right) y, \quad x, y \in H,
$$

$I$ denotes the identity mapping of $H$, and the parameters $\left\{\lambda_{t}\right\},\left\{\beta_{t}^{i}\right\} \subset(0,1)$ for all $t \in(0,1)$ satisfy the following conditions: $\lambda_{t} \rightarrow 0$ as $t \rightarrow 0$ and $0<\liminf _{t \rightarrow 0} \beta_{t}^{i} \leq \lim \sup _{t \rightarrow 0} \beta_{t}^{i}<1, i=$ $1, \ldots, N$. 


\section{Main Result}

We formulate the following facts for the proof of our results.

Lemma 2.1 (see [7]). (i) $\|x+y\|^{2} \leq\|x\|^{2}+2\langle y, x+y\rangle$ and for any fixed $t \in[0,1]$, (ii) $\|(1-t) x+t y\|^{2}=(1-t)\|x\|^{2}+t\|y\|^{2}-(1-t) t\|x-y\|^{2}$, for all $x, y \in H$.

Put $T^{\curlywedge} x=T x-\lambda \mu F(T x), x \in H, \lambda \in[0,1]$; for any nonexpansive mapping $T$ of $H$, we have the following lemma.

Lemma 2.2 (see [8]). $\left\|T^{\curlywedge} x-T^{\curlywedge} y\right\| \leq(1-\lambda \tau)\|x-y\|$, for all $x, y \in H$ and for a fixed number $\mu \in\left(0,2 \eta / L^{2}\right)$, where $\tau=1-\sqrt{1-\mu\left(2 \eta-\mu L^{2}\right)} \in(0,1)$.

Lemma 2.3 (Demiclosedness Principle [9]). Assume that $T$ is a nonexpansive self-mapping of a closed convex subset $K$ of a Hibert space $H$. If $T$ has a fixed point, then $I-T$ is demiclosed; that is, whenever $\left\{x_{k}\right\}$ is a sequence in $K$ weakly converging to some $x \in K$ and the sequence $\left\{(I-T) x_{k}\right\}$ strongly converges to some $y$, it follows that $(I-T) x=y$.

Now, we are in a position to prove the following result.

Theorem 2.4. Let $H$ be a real Hilbert space and $F: H \rightarrow H$ a mapping such that for some constants $L, \eta>0, F$ is L-Lipschitz continuous and $\eta$-strongly monotone. Let $\left\{T_{i}\right\}_{i=1}^{N}$ be $N$ nonexpansive selfmaps of $H$ such that $C=\cap_{i=1}^{N} \operatorname{Fix}\left(T_{i}\right) \neq \emptyset$. Let $\mu \in\left(0,2 \eta / L^{2}\right)$ and let $t \in(0,1),\left\{\lambda_{t}\right\},\left\{\beta_{t}^{i}\right\} \subset(0,1)$, such that

$$
\lambda_{t} \longrightarrow 0, \quad \text { as } t \longrightarrow 0, \quad 0<\lim \inf _{t \rightarrow 0} \beta_{t}^{i} \leq \lim \sup _{t \rightarrow 0} \beta_{t}^{i}<1, \quad i=1, \ldots, N
$$

Then, the net $\left\{x_{t}\right\}$ defined by (1.8)-(1.9) converges strongly to the unique element $p^{*}$ in (1.1).

Proof. By using Lemma 2.2 with $T^{\curlywedge}=T_{0}^{t}$, that is, $T=I$, we have that

$$
\begin{aligned}
\left\|T^{t} x-T^{t} y\right\| & \leq\left(1-\lambda_{t} \tau\right)\left\|T_{N}^{t} \cdots T_{1}^{t} x-T_{N}^{t} \cdots T_{1}^{t} y\right\| \\
& \vdots \\
& \leq\left(1-\lambda_{t} \tau\right)\left\|T_{i}^{t} \cdots T_{1}^{t} x-T_{i}^{t} \cdots T_{1}^{t} y\right\| \\
& \vdots \\
& \leq\left(1-\lambda_{t} \tau\right)\left\|T_{1}^{t} x-T_{1}^{t} y\right\| \leq\left(1-\lambda_{t} \tau\right)\|x-y\| \quad \forall x, y \in H .
\end{aligned}
$$

So, $T^{t}$ is a contraction in $H$. By Banach's Contraction Principle, there exists a unique element $x_{t} \in H$ such that $x_{t}=T^{t} x_{t}$ for all $t \in(0,1)$. 
Next, we show that $\left\{x_{t}\right\}$ is bounded. Indeed, for a fixed point $p \in C$, we have that $T_{i}^{t} p=p$ for $i=1, \ldots, N$, and hence

$$
\begin{aligned}
\left\|x_{t}-p\right\| & =\left\|T^{t} x_{t}-p\right\|=\left\|T^{t} x_{t}-T_{N}^{t} \cdots T_{1}^{t} p\right\| \\
& =\left\|\left(I-\lambda_{t} \mu F\right) T_{N}^{t} \cdots T_{1}^{t} x_{t}-\left(I-\lambda_{t} \mu F\right) T_{N}^{t} \cdots T_{1}^{t} p-\lambda_{t} \mu F(p)\right\| \\
& \leq\left(1-\lambda_{t} \tau\right)\left\|T_{N}^{t} \cdots T_{1}^{t} x_{t}-T_{N}^{t} \cdots T_{1}^{t} p\right\|+\lambda_{t} \mu\|F(p)\| \\
& \leq\left(1-\lambda_{t} \tau\right)\left\|T_{N-1}^{t} \cdots T_{1}^{t} x_{t}-T_{N-1}^{t} \cdots T_{1}^{t} p\right\|+\lambda_{t} \mu\|F(p)\| \\
& \vdots \\
& \leq\left(1-\lambda_{t} \tau\right)\left\|T_{i}^{t} \cdots T_{1}^{t} x_{t}-T_{i}^{t} \cdots T_{1}^{t} p\right\|+\lambda_{t} \mu\|F(p)\| \\
& \vdots \\
& \leq\left(1-\lambda_{t} \tau\right)\left\|T_{1}^{t} x_{t}-T_{1}^{t} p\right\|+\lambda_{t} \mu\|F(p)\| \\
& \leq\left(1-\lambda_{t} \tau\right)\left\|x_{t}-p\right\|+\lambda_{t} \mu\|F(p)\| .
\end{aligned}
$$

Therefore,

$$
\left\|x_{t}-p\right\| \leq \frac{\mu}{\tau}\|F(p)\|
$$

that implies the boundedness of $\left\{x_{t}\right\}$. So, are the nets $\left\{F\left(y_{t}^{N}\right)\right\},\left\{y_{t}^{i}\right\}, i=1, \ldots, N$.

Put

$$
\begin{aligned}
y_{t}^{1} & =\left(1-\beta_{t}^{1}\right) x_{t}+\beta_{t}^{1} T_{1} x_{t} \\
y_{t}^{2} & =\left(1-\beta_{t}^{2}\right) y_{t}^{1}+\beta_{t}^{2} T_{2} y_{t}^{1} \\
& \vdots \\
y_{t}^{i} & =\left(1-\beta_{t}^{i}\right) y_{t}^{i-1}+\beta_{t}^{i} T_{i} y_{t}^{i-1}, \\
& \vdots \\
y_{t}^{N} & =\left(1-\beta_{t}^{N}\right) y_{t}^{N-1}+\beta_{t}^{N} T_{N} y_{t}^{N-1} .
\end{aligned}
$$

Then,

$$
x_{t}=\left(I-\lambda_{t} \mu F\right) y_{t}^{N}
$$


Moreover,

$$
\begin{aligned}
\left\|x_{t}-p\right\|^{2} & =\left\|\left(I-\lambda_{t} \mu F\right) y_{t}^{N}-p\right\|^{2} \\
& =\left\|y_{t}^{N}-p\right\|^{2}-2 \lambda_{t} \mu\left\langle F\left(y_{t}^{N}\right), y_{t}^{N}-p\right\rangle+\lambda_{t}^{2} \mu^{2}\left\|F\left(y_{t}^{N}\right)\right\|^{2} \\
& \leq\left\|y_{t}^{N-1}-p\right\|^{2}-2 \lambda_{t} \mu\left\langle F\left(y_{t}^{N}\right), y_{t}^{N}-p\right\rangle+\lambda_{t}^{2} \mu^{2}\left\|F\left(y_{t}^{N}\right)\right\|^{2} \\
& \vdots \\
& \leq\left\|y_{t}^{1}-p\right\|^{2}-2 \lambda_{t} \mu\left\langle F\left(y_{t}^{N}\right), y_{t}^{N}-p\right\rangle+\lambda_{t}^{2} \mu^{2}\left\|F\left(y_{t}^{N}\right)\right\|^{2} \\
& \leq\left\|x_{t}-p\right\|^{2}-2 \lambda_{t} \mu\left\langle F\left(y_{t}^{N}\right), y_{t}^{N}-p\right\rangle+\lambda_{t}^{2} \mu^{2}\left\|F\left(y_{t}^{N}\right)\right\|^{2} .
\end{aligned}
$$

Thus,

$$
\eta\left\|y_{t}^{N}-p\right\|^{2}+\left\langle F(p), y_{t}^{N}-p\right\rangle \leq \frac{\lambda_{t} \mu}{2}\left\|F\left(y_{t}^{N}\right)\right\|^{2}
$$

Further, for the sake of simplicity, we put $y_{t}^{0}=x_{t}$ and prove that

$$
\left\|y_{t}^{i-1}-T_{i} y_{t}^{i-1}\right\| \longrightarrow 0
$$

as $t \rightarrow 0$ for $i=1, \ldots, N$.

Let $\left\{t_{k}\right\} \subset(0,1)$ be an arbitrary sequence converging to zero as $k \rightarrow \infty$ and $x_{k}:=x_{t_{k}}$. We have to prove that $\left\|y_{k}^{i-1}-T_{i} y_{k}^{i-1}\right\| \rightarrow 0$, where $y_{k}^{i}$ are defined by (2.5) with $t=t_{k}$ and $y_{k}^{i}=y_{t_{k}}^{i}$. Let $\left\{x_{l}\right\}$ be a subsequence of $\left\{x_{k}\right\}$ such that

$$
\lim \sup _{k \rightarrow \infty}\left\|y_{k}^{i-1}-T_{i} y_{k}^{i-1}\right\|=\lim _{l \rightarrow \infty}\left\|y_{l}^{i-1}-T_{i} y_{l}^{i-1}\right\|
$$

Let $\left\{x_{k_{j}}\right\}$ be a subsequence of $\left\{x_{l}\right\}$ such that

$$
\limsup _{k \rightarrow \infty}\left\|x_{k}-p\right\|=\lim _{j \rightarrow \infty}\left\|x_{k_{j}}-p\right\| .
$$


From (2.6) and Lemma 2.1, it implies that

$$
\begin{aligned}
\left\|x_{k_{j}}-p\right\|^{2}= & \left\|\left(I-\lambda_{k_{j}} \mu F\right) y_{k_{j}}^{N}-p\right\|^{2} \\
\leq & \left\|y_{k_{j}}^{N}-p\right\|^{2}-2 \lambda_{k_{j}} \mu\left\langle F\left(y_{k_{j}}^{N}\right), x_{k_{j}}-p\right\rangle \\
= & \left\|\left(1-\beta_{k_{j}}^{N}\right)\left(y_{k_{j}}^{N-1}-p\right)+\beta_{k_{j}}^{N}\left(T_{N} y_{k_{j}}^{N-1}-T_{N} p\right)\right\|^{2} \\
& -2 \lambda_{k_{j}} \mu\left\langle F\left(y_{k_{j}}^{N}\right), x_{k_{j}}-p\right\rangle \\
\leq & \left(1-\beta_{k_{j}}^{N}\right)\left\|y_{k_{j}}^{N-1}-p\right\|^{2}+\beta_{k_{j}}^{N}\left\|T_{N} y_{k_{j}}^{N-1}-T_{N} p\right\|^{2} \\
& -2 \lambda_{k_{j}} \mu\left\langle F\left(y_{k_{j}}^{N}\right), x_{k_{j}}-p\right\rangle \\
\leq & \left\|y_{k_{j}}^{N-1}-p\right\|^{2}-2 \lambda_{k_{j}} \mu\left\langle F\left(y_{k_{j}}^{N}\right), x_{k_{j}}-p\right\rangle \\
\leq & \cdots \leq\left\|y_{k_{j}}^{1}-p\right\|^{2}-2 \lambda_{k_{j}} \mu\left\langle F\left(y_{k_{j}}^{N}\right), x_{k_{j}}-p\right\rangle \\
\leq & \left\|x_{k_{j}}-p\right\|^{2}-2 \lambda_{k_{j}} \mu\left\langle F\left(y_{k_{j}}^{N}\right), x_{k_{j}}-p\right\rangle .
\end{aligned}
$$

Hence,

$$
\lim _{j \rightarrow \infty}\left\|x_{k_{j}}-p\right\|=\lim _{j \rightarrow \infty}\left\|y_{k_{j}}^{i}-p\right\|, \quad i=1, \ldots, N
$$

By Lemma 2.1,

$$
\begin{aligned}
\left\|y_{k_{j}}^{i}-p\right\|^{2}= & \left(1-\beta_{k_{j}}^{i}\right)\left\|y_{k_{j}}^{i-1}-p\right\|^{2}+\beta_{k_{j}}^{i}\left\|T_{i} y_{k_{j}}^{i-1}-p\right\|^{2} \\
& -\beta_{k_{j}}^{i}\left(1-\beta_{k_{j}}^{i}\right)\left\|y_{k_{j}}^{i-1}-T_{i} y_{k_{j}}^{i-1}\right\|^{2} \\
\leq & \left(1-\beta_{k_{j}}^{i}\right)\left\|y_{k_{j}}^{i-1}-p\right\|^{2}+\beta_{k_{j}}^{i}\left\|y_{k_{j}}^{i-1}-p\right\|^{2} \\
& -\beta_{k_{j}}^{i}\left(1-\beta_{k_{j}}^{i}\right)\left\|y_{k_{j}}^{i-1}-T_{i} y_{k_{j}}^{i-1}\right\|^{2} \\
= & \left\|y_{k_{j}}^{i-1}-p\right\|^{2}-\beta_{k_{j}}^{i}\left(1-\beta_{k_{j}}^{i}\right)\left\|y_{k_{j}}^{i-1}-T_{i} y_{k_{j}}^{i-1}\right\|^{2} \\
\leq & \cdots=\left\|y_{k_{j}}^{0}-p\right\|^{2}-\beta_{k_{j}}^{i}\left(1-\beta_{k_{j}}^{i}\right)\left\|y_{k_{j}}^{i-1}-T_{i} y_{k_{j}}^{i-1}\right\|^{2} \\
= & \left\|x_{k_{j}}-p\right\|^{2}-\beta_{k_{j}}^{i}\left(1-\beta_{k_{j}}^{i}\right)\left\|y_{k_{j}}^{i-1}-T_{i} y_{k_{j}}^{i-1}\right\|^{2}, \quad i=1, \ldots, N .
\end{aligned}
$$


Without loss of generality, we can assume that $\alpha \leq \beta_{t}^{i} \leq \beta$ for some $\alpha, \beta \in(0,1)$. Then, we have

$$
\alpha(1-\beta)\left\|y_{k_{j}}^{i-1}-T_{i} y_{k_{j}}^{i-1}\right\|^{2} \leq\left\|x_{k_{j}}-p\right\|^{2}-\left\|y_{k_{j}}^{i}-p\right\|^{2}
$$

This together with (2.13) implies that

$$
\lim _{j \rightarrow \infty}\left\|y_{k_{j}}^{i-1}-T_{i} y_{k_{j}}^{i-1}\right\|^{2}=0, \quad i=1, \ldots, N
$$

It means that $\left\|y_{t}^{i-1}-T_{i} y_{t}^{i-1}\right\| \rightarrow 0$ as $t \rightarrow 0$ for $i=1, \ldots, N$.

Next, we show that $\left\|x_{t}-T_{i} x_{t}\right\| \rightarrow 0$ as $t \rightarrow 0$. In fact, in the case that $i=1$ we have $y_{t}^{0}=x_{t}$. So, $\left\|x_{t}-T_{1} x_{t}\right\| \rightarrow 0$ as $t \rightarrow 0$. Further, since

$$
\left\|y_{t}^{1}-T_{1} x_{t}\right\|=\left(1-\beta_{t}^{1}\right)\left\|x_{t}-T_{1} x_{t}\right\|
$$

and $\left\|x_{t}-T_{1} x_{t}\right\| \rightarrow 0$, we have that $\left\|y_{t}^{1}-T_{1} x_{t}\right\| \rightarrow 0$. Therefore, from

$$
\left\|x_{t}-y_{t}^{1}\right\| \leq\left\|x_{t}-T_{1} x_{t}\right\|+\left\|T_{1} x_{t}-y_{t}^{1}\right\|
$$

it follows that $\left\|x_{t}-y_{t}^{1}\right\| \rightarrow 0$ as $t \rightarrow 0$. On the other hand, since

$$
\begin{aligned}
\left\|y_{t}^{2}-T_{2} y_{t}^{1}\right\| & =\left(1-\beta_{t}^{2}\right)\left\|y_{t}^{1}-T_{2} y_{t}^{1}\right\| \longrightarrow 0, \\
\left\|y_{t}^{2}-x_{t}\right\| & \leq\left(1-\beta_{t}^{2}\right)\left\|y_{t}^{1}-x_{t}\right\|+\beta_{t}^{2}\left\|T_{2} y_{t}^{1}-x_{t}\right\| \\
& \leq\left(1-\beta_{t}^{2}\right)\left\|y_{t}^{1}-x_{t}\right\|+\beta_{t}^{2}\left\|T_{2} y_{t}^{1}-y_{t}^{1}\right\|+\left\|y_{t}^{1}-x_{t}\right\|,
\end{aligned}
$$

we obtain that $\left\|y_{t}^{2}-x_{t}\right\| \rightarrow 0$ as $t \rightarrow 0$. Now, from

$$
\begin{aligned}
\left\|x_{t}-T_{2} x_{t}\right\| & \leq\left\|x_{t}-y_{t}^{2}\right\|+\left\|y_{t}^{2}-T_{2} y_{t}^{1}\right\|+\left\|T_{2} y_{t}^{1}-T_{2} x_{t}\right\| \\
& \leq\left\|x_{t}-y_{t}^{2}\right\|+\left\|y_{t}^{2}-T_{2} y_{t}^{1}\right\|+\left\|y_{t}^{1}-x_{t}\right\|,
\end{aligned}
$$

and $\left\|x_{t}-y_{t}^{2}\right\|,\left\|y_{t}^{2}-T_{2} y_{t}^{1}\right\|,\left\|y_{t}^{1}-x_{t}\right\| \rightarrow 0$, it follows that $\left\|x_{t}-T_{2} x_{t}\right\| \rightarrow 0$. Similarly, we obtain that $\left\|x_{t}-T_{i} x_{t}\right\| \rightarrow 0$, for $i=1, \ldots, N$ and $\left\|y_{t}^{N}-x_{t}\right\| \rightarrow 0$ as $t \rightarrow 0$.

Let $\left\{x_{k}\right\}$ be any sequence of $\left\{x_{t}\right\}$ converging weakly to $\tilde{p}$ as $k \rightarrow \infty$. Then, $\| x_{k}-$ $T_{i} x_{k} \| \rightarrow 0$, for $i=1, \ldots, N$ and $\left\{y_{k}^{N}\right\}$ also converges weakly to $\tilde{p}$. By Lemma 2.3, we have $\tilde{p} \in C=\cap_{i=1}^{N} \operatorname{Fix}\left(T_{i}\right)$ and from (2.8), it follows that

$$
\langle F(p), p-\tilde{p}\rangle \geq 0 \quad \forall p \in C .
$$


Since $p, \tilde{p} \in C$, by replacing $p$ by $t p+(1-t) \tilde{p}$ in the last inequality, dividing by $t$ and taking $t \rightarrow 0$ in the just obtained inequality, we obtain

$$
\langle F(\tilde{p}), p-\tilde{p}\rangle \geq 0 \quad \forall p \in C .
$$

The uniqueness of $p^{*}$ in (1.1) guarantees that $\tilde{p}=p^{*}$. Again, replacing $p$ in (2.8) by $p^{*}$, we obtain the strong convergence for $\left\{x_{t}\right\}$. This completes the proof.

\section{Application}

Recall that a mapping $S: H \rightarrow H$ is called a $\gamma$-strictly pseudocontractive if there exists a constant $\gamma \in[0,1)$ such that

$$
\|S x-S y\|^{2} \leq\|x-y\|^{2}+\gamma\|(I-S) x-(I-S) y\|^{2}, \quad \forall x, y \in H
$$

It is well known [10] that a mapping $T: H \rightarrow H$ by $T x=\alpha x+(1-\alpha) S x$ with a fixed $\alpha \in[\gamma, 1)$ for all $x \in H$ is a nonexpansive mapping and $\operatorname{Fix}(T)=\operatorname{Fix}(S)$. Using this fact, we can extend our result to the case $C=\cap_{i=1}^{N} \operatorname{Fix}\left(S_{i}\right)$, where $S_{i}$ is $\gamma_{i}$-strictly pseudocontractive as follows.

Let $\alpha_{i} \in\left[\gamma_{i}, 1\right)$ be fixed numbers. Then, $C=\cap_{i=1}^{N} \operatorname{Fix}\left(\widetilde{T}_{i}\right)$ with $\widetilde{T}_{i} y=\alpha_{i} y+\left(1-\alpha_{i}\right) S_{i} y$, a nonexpansive mapping, for $i=1, \ldots, N$, and hence

$$
\begin{aligned}
\widetilde{T}_{i}^{t} y & =\left(1-\beta_{t}^{i}\right) y+\beta_{t}^{i} \widetilde{T}_{i} y \\
& =\left(1-\beta_{t}^{i}\left(1-\alpha_{i}\right)\right) y+\beta_{t}^{i}\left(1-\alpha_{i}\right) S_{i} y, \quad i=1, \ldots, N
\end{aligned}
$$

So, we have the following result.

Theorem 3.1. Let $H$ be a real Hilbert space and $F: H \rightarrow H$ a mapping such that for some constants $L, \eta>0, F$ is L-Lipschitz continuous and $\eta$-strongly monotone. Let $\left\{S_{i}\right\}_{i=1}^{N}$ be $N \gamma_{i}$-strictly pseudocontractive self-maps of $H$ such that $C=\cap_{i=1}^{N} \operatorname{Fix}\left(S_{i}\right) \neq \emptyset$. Let $\alpha_{i} \in\left[\gamma_{i}, 1\right), \mu \in\left(0,2 \eta / L^{2}\right)$ and let $t \in(0,1),\left\{\lambda_{t}\right\},\left\{\beta_{t}^{i}\right\} \subset(0,1)$, such that

$$
\lambda_{t} \longrightarrow 0, \quad \text { as } t \longrightarrow 0, \quad 0<\lim \inf _{t \rightarrow 0} \beta_{t}^{i} \leq \lim \sup _{t \rightarrow 0} \beta_{t}^{i}<1, \quad i=1, \ldots, N
$$

Then, the net $\left\{x_{t}\right\}$ defined by

$$
x_{t}=\tilde{T}^{t} x_{t}, \quad \tilde{T}^{t}:=T_{0}^{t} \tilde{T}_{N}^{t} \cdots \tilde{T}_{1}^{t}, \quad t \in(0,1),
$$

where $\tilde{T}_{i}^{t}$, for $i=1, \ldots, N$, are defined by (3.2) and $T_{0}^{t} x=\left(I-\lambda_{t} \mu F\right) x$, converges strongly to the unique element $p^{*}$ in (1.1).

It is known in [11] that $\operatorname{Fix}(\widetilde{S})=C$ where $\widetilde{S}=\sum_{i=1}^{N} \xi_{i} S_{i}$ with $\xi_{i}>0$ and $\sum_{i=1}^{N} \xi_{i}=1$ for $N \gamma_{i}$-strictly pseudocontractions $\left\{S_{i}\right\}_{i=1}^{N}$. Moreover, $\widetilde{S}$ is $\gamma$-strictly pseudocontractive with $\gamma=\max \left\{\gamma_{i}: 1 \leq i \leq N\right\}$. So, we also have the following result. 
Theorem 3.2. Let $H$ be a real Hilbert space and $F: H \rightarrow H$ a mapping such that for some constants $L, \eta>0, F$ is L-Lipschitz continuous and $\eta$-strongly monotone. Let $\left\{S_{i}\right\}_{i=1}^{N}$ be $N \gamma_{i^{-}}$ strictly pseudocontractive self-maps of $H$ such that $C=\cap_{i=1}^{N} \operatorname{Fix}\left(S_{i}\right) \neq \emptyset$. Let $\alpha \in[\gamma, 1)$, where $\gamma=\max \left\{\gamma_{i}: 1 \leq i \leq N\right\}, \mu \in\left(0,2 \eta / L^{2}\right)$, and let $t \in(0,1),\left\{\lambda_{t}\right\},\left\{\beta_{t}\right\} \subset(0,1)$, such that

$$
\lambda_{t} \longrightarrow 0, \quad \text { as } t \longrightarrow 0, \quad 0<\lim \inf _{t \rightarrow 0} \beta_{t} \leq \limsup \sup _{t \rightarrow 0} \beta_{t}<1
$$

Then, the net $\left\{x_{t}\right\}$, defined by

$$
x_{t}=\tilde{T}^{t} x_{t}, \quad \tilde{T}^{t}:=T_{0}^{t}\left(\left(1-\beta_{t}(1-\alpha)\right) I+\beta_{t}(1-\alpha) \sum_{i=1}^{N} \xi_{i} S_{i}\right), \quad t \in(0,1),
$$

where $T_{0}^{t}=\left(I-\lambda_{t} \mu F\right), \xi_{i}>0$, and $\sum_{i=1}^{N} \xi_{i}=1$, converges strongly to the unique element $p^{*}$ in (1.1).

\section{Acknowledgment}

This work was supported by the Vietnamese National Foundation of Science and Technology Development.

\section{References}

[1] D. Kinderlehrer and G. Stampacchia, An Introduction to Variational Inequalities and Their Applications, vol. 88 of Pure and Applied Mathematics, Academic Press, New York, NY, USA, 1980.

[2] R. Glowinski, Numerical Methods for Nonlinear Variational Problems, Springer Series in Computational Physics, Springer, New York, NY, USA, 1984.

[3] E. Zeidler, Nonlinear Functional Analysis and Its Applications. III, Springer, New York, NY, USA, 1985.

[4] H.-K. Xu and R. G. Ori, "An implicit iteration process for nonexpansive mappings," Numerical Functional Analysis and Optimization, vol. 22, no. 5-6, pp. 767-773, 2001.

[5] L.-C. Zeng and J.-C. Yao, "Implicit iteration scheme with perturbed mapping for common fixed points of a finite family of nonexpansive mappings," Nonlinear Analysis. Theory, Methods \& Applications, vol. 64, no. 11, pp. 2507-2515, 2006.

[6] L.-C. Ceng, N.-C. Wong, and J.-C. Yao, "Fixed point solutions of variational inequalities for a finite family of asymptotically nonexpansive mappings without common fixed point assumption," Computers E Mathematics with Applications, vol. 56, no. 9, pp. 2312-2322, 2008.

[7] G. Marino and H.-K. Xu, "Weak and strong convergence theorems for strict pseudo-contractions in Hilbert spaces," Journal of Mathematical Analysis and Applications, vol. 329, no. 1, pp. 336-346, 2007.

[8] Y. Yamada, "The hybrid steepest-descent method for variational inequalities problems over the intesectionof the fixed point sets of nonexpansive mappings," in Inhently Parallel Algorithms in Feasibility and Optimization and Their Applications, D. Butnariu, Y. Censor, and S. Reich, Eds., pp. 473504, North-Holland, Amsterdam, Holland, 2001.

[9] K. Goebel and W. A. Kirk, Topics in Metric Fixed Point Theory, vol. 28 of Cambridge Studies in Advanced Mathematics, Cambridge University Press, Cambridge, UK, 1990.

[10] H. Zhou, "Convergence theorems of fixed points for $\kappa$-strict pseudo-contractions in Hilbert spaces," Nonlinear Analysis. Theory, Methods \& Applications, vol. 69, no. 2, pp. 456-462, 2008.

[11] G. L. Acedo and H.-K. Xu, "Iterative methods for strict pseudo-contractions in Hilbert spaces," Nonlinear Analysis. Theory, Methods E Applications, vol. 67, no. 7, pp. 2258-2271, 2007. 